Artículo

\title{
Osmoacondicionamiento de maíces criollos: efectos sobre la fenología y crecimiento
}

Fernando Mejía Ramírez ${ }^{1}$

Mepivoseth Castelán Estrada ${ }^{1 \S}$

Luz del Carmen Lagunes Espinoza ${ }^{1}$

José Jesús Obrador Olán ${ }^{1}$

Francisco Marcelo Lara Viveros ${ }^{2}$

${ }^{1}$ Posgrado en Producción Agroalimentaria en el Trópico-Colegio de Postgraduados-Campus Tabasco. Cárdenas, Tabasco. AP. 24. CP. 86500. (mejia.fernando@colpos.mx; lagunesc@colpos.mx; obradoro@colpos.mx). ${ }^{2}$ Universidad Politécnica de Francisco I. Madero. Tepatepec, Hidalgo. CP. 42660. (fmlara@upfim.edu.mx).

${ }^{\S}$ Autor para correspondencia: mcastelan@ colpos.mx.

\section{Resumen}

Dada la importancia de los maíces criollos para los pequeños agricultores de México y la necesidad de aprovechar la humedad en los cultivos de temporal, el objetivo de este trabajo fue estudiar los efectos de tratamientos de osmoacondicionamiento en semillas de maíz sobre la emergencia, crecimiento y fenología de las plantas resultantes. Los tratamientos fueron agua y una solución de $\mathrm{AG}_{3}$ a $0.3 \mathrm{~g} \mathrm{~L}^{-1}$ con dos tiempos de exposición (12 y $18 \mathrm{~h}$ ) en maíces criollos (Mején, Chalqueño y Negrito) y una variedad comercial (VS-536). Las variables observadas fueron: porcentaje y velocidad de emergencia, crecimiento, materia seca, días a floración y concentración NPK. El diseño experimental en bloques completos al azar con arreglo factorial ( $4 \times 2 \times 2)$ tuvo cuatro repeticiones y cuatro testigos. Los resultados mostraron que el factor variedad fue altamente significativo $(p \leq 0.001)$ para el porcentaje emergencia, donde el criollo Negrito con $12 \mathrm{~h}$ mostró el valor más alto (76.8\%) 3 días después de la siembra (dds), aunque el Mején mostró mayor velocidad de emergencia (4.6 semillas día ${ }^{-1}$ y mayor tasa de crecimiento $\left(4.32 \mathrm{~cm} \mathrm{día}^{-1}\right.$ planta $\left.^{-1}\right)$ a los 11 dds. Por otra parte, el criollo Chalqueño alcanzó la mayor ganancia de materia seca $(0.71 \mathrm{~g}$ día $^{-1}$ planta $^{-1}$ ) a los $25 \mathrm{dds}$, mientras que el Mején mostró menor número de días a floración masculina y femenina (48 y 55, respectivamente). La mayor concentración de NPK en planta se encontró en la variedad VS-536. Se concluye que los tratamientos de osmoacondicionamiento inducen efectos positivos sobre la emergencia, crecimiento y fenología de los maíces estudiados.

Palabras clave: altura, emergencia, floración, imbibición.

Recibido: octubre de 2019

Aceptado: diciembre de 2019 


\section{Introducción}

El maíz (Zea mays L.) es el cultivo agrícola más importante en México donde $82 \%$ de las siembras se realizan bajo condiciones de temporal. Para este cultivo $75 \%$ de los productores emplean semillas de razas nativas y cada productor cultiva de una a tres razas diferentes (Espinosa-Carderón et al., 2017). Los maíces nativos presentan adaptabilidad a condiciones agroecológicas locales, lo que se manifiesta en diferentes niveles de precocidad y resistencia a factores adversos (ÁngelesGaspar et al., 2010), además que las razas nativas requieren menor cantidad de insumos para su cultivo.

Sin embargo, en condiciones de temporal estas semillas presentarían baja germinación y mayor número de días a la emergencia, lo que provoca poca uniformidad y bajo aprovechamiento del agua, razón que hace necesario buscar opciones que contribuyan a superar estas limitantes. Una alternativa es el osmoacondicionamiento de las semillas el cual es un método probado que mejora la calidad fisiológica de la semilla para lograr uniformidad de emergencia y mayor porcentaje de germinación. El objetivo del presente trabajo fue evaluar el efecto de diferentes tratamientos de osmoacondicionamiento de semillas sobre la emergencia, crecimiento, fenología y concentración NPK de maíces criollos, en condiciones de invernadero.

\section{Materiales y métodos}

El trabajo se realizó en el invernadero del Campo Experimental del Colegio de Posgraduados Campus Tabasco, localizado en las coordenadas geográficas $17^{\circ} 59^{\prime} 10^{\prime}$ ' latitud norte y $93^{\circ} 35^{\prime}$ 02 " longitud oeste, a una altitud de $18 \mathrm{~m}$. Para el osmoacondicionamiento, 80 semillas de maíz por cada variedad fueron sometidas a tratamiento (agua o solución de $\mathrm{AG}_{3}$ en conc. de $0.3 \mathrm{~g} \mathrm{~L}^{-1}$ ) durante dos tiempos (12 y $18 \mathrm{~h}$ ), más un testigo por cada tratamiento, con cuatro repeticiones (20 tratamientos en total). Se utilizaron tres razas criollas de maíz (Negrito y Chalqueño del estado de Hidalgo y Mején del estado de Tabasco) y la variedad sintética VS-536. Las semillas de cada variedad se osmoacondicionaron en la solución y tiempos establecidos; al término, las semillas se retiraron para airearlas durante $48 \mathrm{~h}$ a temperatura ambiente.

La siembra se realizó posterior a los tratamientos en contenedores plásticos con capacidad de 7.5 L llenados con el sustrato suelo: cachaza de caña de azúcar $(1: 1 \mathrm{v} / \mathrm{v})$. El diseño experimental fue completamente al azar con arreglo factorial 4 × 2 × 2 (cuatro tipos de maíz, dos soluciones osmóticas y dos tiempos de imbibición) con cuatro repeticiones. Cada tratamiento constó de 80 plantas en total.

\section{Porcentaje de emergencia}

El número de plántulas emergidas fue contabilizado diariamente, considerando como emergencia el momento que la plántula fue visible. Los conteos se hicieron desde uno y hasta cinco días despúes de la siembra (dds), tiempo en que se alcanzó 100\% de emergencia en las variedades de maíz evaluadas. Los cálculos se realizaron con la fórmula (Ahammad et al., 2014).

$\%$ de plántulas emergidas $=\frac{\text { Número de plántulas emergidas }}{\text { Número de semillas sembradas }} \times 100$ 


\section{Velocidad de emergencia (semillas día ${ }^{-1}$ )}

A partir de los datos de emergencia y días se calculó la velocidad de emergencia (semillas por día) para cada variedad, con la siguiente fórmula (Maguire, 1962).

$\mathrm{VE}=\sum \mathrm{ni} / \mathrm{t}$

Donde: $\mathrm{VE}=$ velocidad de emergencia, $\mathrm{ni}=$ número de semillas emergidas; $\mathrm{t}=$ tiempo de emergencia (días).

\section{Tasa absoluta de crecimiento}

A partir de los datos de altura de planta y ganancia de biomasa se calculó la tasa absoluta de crecimiento (TAC) expresada como altura $\left(\mathrm{cm} \mathrm{día}{ }^{-1}\right.$ planta $\left.^{-1}\right)$ y materia seca $\left(\mathrm{g} \mathrm{día}^{-1}\right.$ planta $\left.^{-1}\right)$, mediante la fórmula (Barrera et al., 2010).

$\mathrm{TAC}=\frac{\mathrm{P} 2-\mathrm{P} 1}{\mathrm{~T} 2-\mathrm{T} 1}$

Donde: TAC $=$ tasa absoluta de crecimiento $\mathrm{P} 1=$ altura $\mathrm{o}$ materia seca inicial; $\mathrm{P} 2=$ altura $\mathrm{o}$ materia seca final; $\mathrm{T} 1=$ tiempo inicial; $\mathrm{T} 2=$ tiempo final.

\section{Días a floración masculina y femenina}

Se registró el número de días transcurridos desde la siembra y hasta el momento en que 50\% de las plantas presentaron su floración, tanto masculina como femenina.

\section{Concentración de NPK}

Para determinar el posible efecto del osmoacondicionamiento sobre la concentración de nitrógeno, fósforo y potasio en planta, se muestrearon plantas completas a los 59 dds. Estas fueron secadas a $60{ }^{\circ} \mathrm{C}$ durante $48 \mathrm{~h}$. Las muestras compuestas (repeticiones) se molieron y se enviaron al laboratorio para el análisis elemental NPK.

\section{Análisis estadístico}

Para obtener una distribución normalizada de los datos se hizo la trasformación logarítmica [T= $\log (\mathrm{y})$ ]. Después se hicieron los análisis de varianza bajo un diseño factorial, así como las pruebas de medias (Tukey $p \leq 0.05$ ). Para los análisis estadísticos se utilizó el programa SAS (versión 9.4).

\section{Resultados y discusión}

\section{Porcentaje de emergencia}

El análisis de varianza para las variables evaluadas muestra un efecto altamente significativo ( $p \leq$ 0.001 ) del factor variedad sobre el porcentaje de emergencia, velocidad de emergencia, altura y acumulación de materia seca de la planta, mientras que para los días a floración femenina $(p \leq 0.01)$ 
y masculina ( $p \leq 0.05$ ) el efecto fue significativo. Por su parte, el efecto del factor solución fue significativo para días a floración femenina $(p \leq 0.05)$. La interacción raza*tiempo fue significativa para altura de planta $(p \leq 0.001)$ y significativa para porcentaje de emergencia $(p \leq 0.01)$ mientras que la triple interacción solo fue significativa $(p \leq 0.01)$ para días a floración masculina (Cuadro 1).

Cuadro 1. Análisis de varianza de las variables en estudio, para maíces bajo tratamientos de osmoacondicionamiento.

\begin{tabular}{|c|c|c|c|c|c|c|}
\hline \multirow{2}{*}{$\begin{array}{l}\text { Factor de } \\
\text { variación }\end{array}$} & \multirow{2}{*}{$\mathrm{PE}$} & \multirow{2}{*}{$\begin{array}{c}\text { VE } \\
\left(\text { semillas día }{ }^{-1}\right)\end{array}$} & \multicolumn{2}{|c|}{ Floración (días) } & \multirow{2}{*}{$\begin{array}{c}\text { Materia seca } \\
\left(\mathrm{g} \mathrm{día}^{-1} \text { planta }^{-1}\right)\end{array}$} & \multirow{2}{*}{$\begin{array}{c}\text { Crecimiento } \\
\left(\mathrm{cm} \mathrm{día}^{-1} \text { planta }^{-1}\right)\end{array}$} \\
\hline & & & $\hat{\sigma}$ & $q$ & & \\
\hline Raza (R) & $1.3^{* * *}$ & $1.2^{* *}$ & $53.1^{*}$ & $60.5^{* *}$ & $0.56^{* *}$ & $7.26^{*}$ \\
\hline Tiempo (T) & 0.07 & 0.04 & 25 & 3.5 & 0.01 & 0.06 \\
\hline Solución (S) & 0.01 & 0.03 & 18 & $50.7^{*}$ & 0.22 & 0.02 \\
\hline $\mathrm{R} * \mathrm{~T}$ & $0.14^{* *}$ & 0.29 & 26.3 & 8.5 & 0.52 & 1.74 \\
\hline $\mathrm{R} * \mathrm{~S}$ & 0.01 & 0.03 & 9.7 & 4.6 & 0.11 & 1.13 \\
\hline $\mathrm{T} * \mathrm{~S}$ & 0.07 & 0.12 & 4 & 15 & $0.57^{*}$ & 6 \\
\hline $\mathrm{R} * \mathrm{~T} * \mathrm{~S}$ & 0.02 & 0.35 & $65.1^{* * *}$ & 18.6 & 0.02 & 0.98 \\
\hline Error & 5.8 & 13.44 & 779.75 & 667.75 & 20.71 & 654.75 \\
\hline $\mathrm{CV}$ & 9.94 & 11.18 & 11 & 5.7 & 30.29 & 29.81 \\
\hline
\end{tabular}

${ }^{*} ;{ }^{* *} ;{ }^{* * *}=$ diferencia significativa con $p \leq 0.05, p \leq 0.01, p \leq 0.001$, respectivamente. $\mathrm{PE}=$ porcentaje de emergencia; $\mathrm{VE}=$ velocidad de emergencia.

Los criollos Negrito y Chalqueño presentaron una emergencia más rápida en comparación con Mején y la VS-536, obteniendo Negrito 74\% de emergencia a los 3 dds y Chalqueño el 50\% (Figura 1). Conforme avanzó el tiempo, la emergencia de estos maíces cambió y a los 7 dds Mején, Negrito y VS-536 alcanzaron mayores porcentajes de emergencia que Chalqueño (89.6\%). Estas variedades con mayor porcentaje de emergencia en menor tiempo presentan una característica favorable, ya que al emerger más rápido las plántulas logran un crecimiento aéreo y radical para el establecimiento del cultivo (Imran et al., 2013). Estos autores al probar varios tratamientos encontraron que los maíces osmoacondicionados presentaron mayor cantidad de raíces.

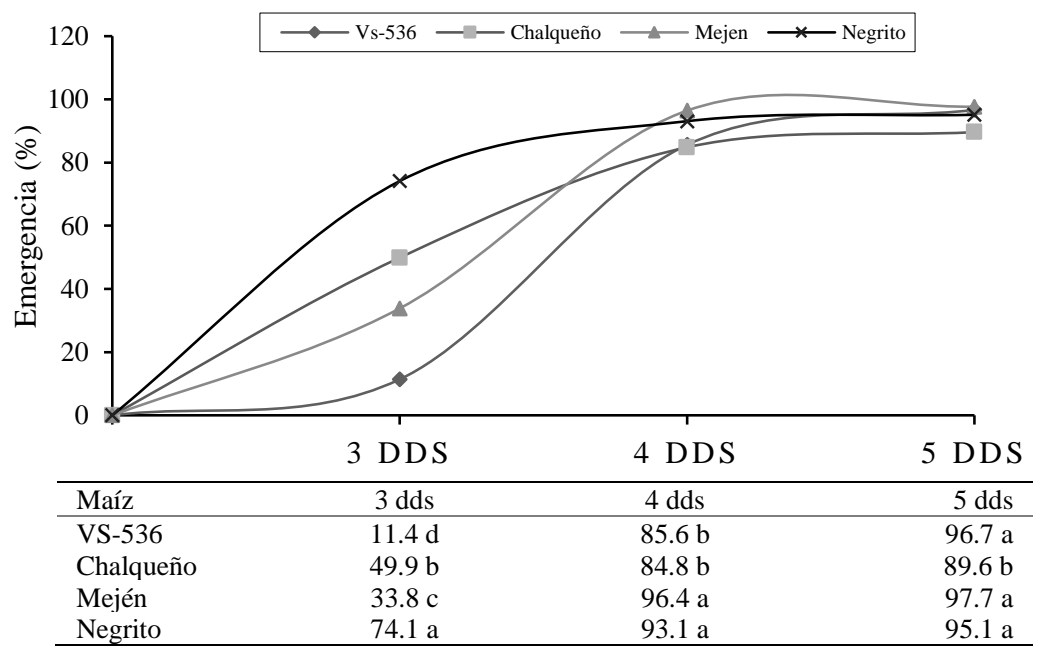

Figura 1. Efecto de tratamientos de osmoacondicionamiento sobre el porcentaje de emergencia en maíces criollos y una variedad sintética. 
A los 5 dds el maíz criollo Mején obtuvo 97.7\% de emergencia, lo que favorece mayor número de plantas por hectárea y potencialmente mejor rendimiento del cultivo (Tonaka y Yamaguchi, 2014). En Tabasco se ha reportado que los mejores rendimientos de maíz se obtienen en densidades de 60 000 y 70000 plantas ha $^{-1}$ (De la Cruz-Lazaro et al., 2009) razón por la que es importante evitar los bajos porcentajes de emergencia para que los rendimientos de maíz no se vean disminuidos (Barrón-Freyre, 2008).

\section{Interacción variedad*tiempo}

A los 3 dds se observaron diferencias significativas entre los criollos Negrito, Chalqueño y Mején con $18 \mathrm{~h}$ de osmoacondicionamiento, que presentaron valores superiores al resto de tratamientos. El criollo Negrito mostró el mayor porcentaje de emergencia $(>70 \%)$ durante este periodo de observación (Cuadro 2).

Cuadro 2. Efecto de la interacción raza*tiempo de osmoacondicionamiento sobre el porcentaje de emergencia de semillas de maíz.

\begin{tabular}{cccc}
\hline Maíz*tiempo & $3 \mathrm{dds}$ & $4 \mathrm{dds}$ & $5 \mathrm{dds}$ \\
\hline Negrito 12 h & $76.8 \pm 10.4 \mathrm{a}$ & $93.7 \pm 4.4 \mathrm{ab}$ & $96.2 \pm 3.5 \mathrm{abc}$ \\
Negrito testigo & $76.8 \pm 2.58 \mathrm{a}$ & $94.9 \pm 5.7 \mathrm{ab}$ & $97.5 \pm 2.8 \mathrm{abc}$ \\
Negrito 18 h & $70.3 \pm 7.3 \mathrm{a}$ & $91.6 \pm 7 \mathrm{ab}$ & $92.9 \pm 6.5 \mathrm{abc}$ \\
Chalqueño testigo & $57 \pm 8.6 \mathrm{a}$ & $89.5 \pm 10.8 \mathrm{ab}$ & $94.8 \pm 7 \mathrm{abc}$ \\
Chalqueño 12 h & $55.6 \pm 13.1 \mathrm{a}$ & $86.1 \pm 5.2 \mathrm{ab}$ & $88.5 \pm 6.4 \mathrm{bc}$ \\
Mején 18 h & $44.1 \pm 12.7 \mathrm{a}$ & $98.1 \pm 3.7 \mathrm{a}$ & $98.1 \pm 3.7 \mathrm{abc}$ \\
Chalqueño 18 h & $41.9 \pm 13.7 \mathrm{a}$ & $81.4 \pm 10 \mathrm{~b}$ & $88.2 \pm 10.3 \mathrm{c}$ \\
Mején 12 h & $36.5 \pm 19 \mathrm{ab}$ & $96.8 \pm 2.6 \mathrm{a}$ & $99.4 \pm 1.8 \mathrm{ab}$ \\
VS 536 18 h & $18.6 \pm 8.5 \mathrm{bc}$ & $84.6 \pm 8.5 \mathrm{ab}$ & $95.5 \pm 4.2 \mathrm{abc}$ \\
Mején testigo & $17 \pm 18.8 \mathrm{bc}$ & $92.5 \pm 2.8 \mathrm{ab}$ & $93.7 \pm 4.7 \mathrm{abc}$ \\
VS 536 12 h & $8.8 \pm 8.8 \mathrm{~cd}$ & $81.7 \pm 12 \mathrm{~b}$ & $96.2 \pm 4.4 \mathrm{abc}$ \\
VS-536 testigo & $7.1 \pm 5.77 \mathrm{~d}$ & $96.2 \pm 4.79 \mathrm{ab}$ & $100 \pm 0 \mathrm{a}$ \\
\hline
\end{tabular}

Letras diferentes dentro de columnas, indican diferencia estadística significativa (Tukey $\mathrm{p} \leq 0.05$ ); dds $=$ días después de la siembra.

A los 5 dds el testigo VS-536 obtuvo 100\% de emergencia, seguido del tratamiento Mején $12 \mathrm{~h}$ (99.4\%), mientras que el Chalqueño 18 h presentó la emergencia más baja (88\%); no obstante, este porcentaje de emergencia es superior a lo reportado por Nicasio-Arzeta et al. (2011) en maíz Chalqueño osmoacondicionado por $24 \mathrm{~h}$. En trabajos previos Afzal et al. (2002) reportan mayor porcentaje de germinación en semillas de maíz osmoacondicionadas por $24 \mathrm{~h}$, mientras que Moradi et al. (2008) indican un mejor porcentaje de germinación en un tiempo de $36 \mathrm{~h}$. Por su parte, Rangel-Fajardo et al. (2014) indican que, después de un punto dado, a mayor tiempo de osmoacondicionamiento el porcentaje de emergencia tiende a disminuir. 


\section{Velocidad de emergencia}

La velocidad de emergencia fue estadísticamente superior en el criollo Mején ya que presentó una tasa de 4.6 semillas día $^{-1}$ a los 5 dds mientras que el Chalqueño tuvo una velocidad de 4 semillas día $^{-1}$ (Figura 2). La mayor velocidad de emergencia favorece el establecimiento del cultivo evitando pérdidas y permitiendo mayor uniformidad, así como mayor altura de planta, en menor tiempo (Imran et al., 2013; Karmore y Tomar, 2015).

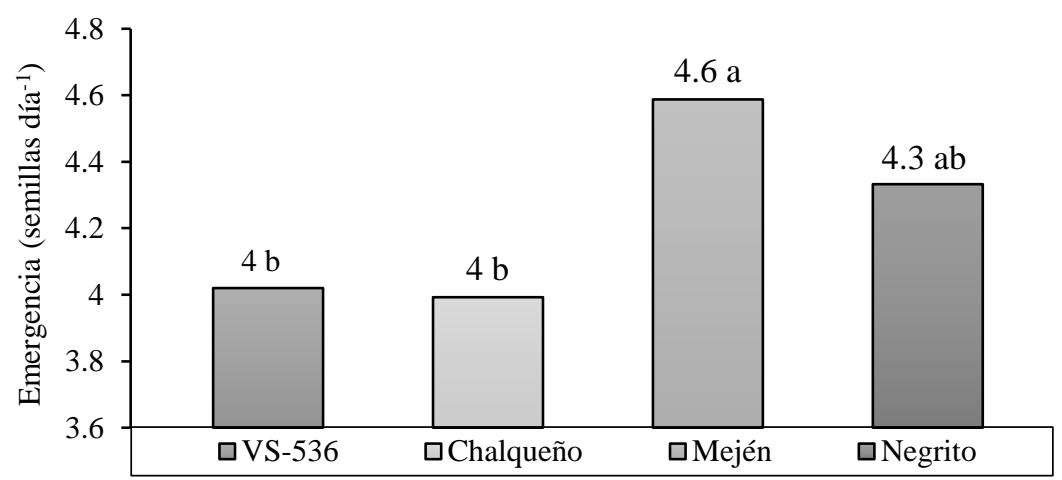

Figura 2. Velocidad de emergencia de semillas de maíz bajo tratamientos de osmoacondicionamiento, cinco días después de la siembra.

\section{Tasa absoluta de crecimiento en altura}

Aunque inicialmente el criollo Mején presentó mayor tasa de crecimiento que las otras variedades (4.32 $\mathrm{cm} \mathrm{día}^{-1}$ planta $^{-1}$ ), hacia el final del periodo de observaciones (46 dds) se encontró por debajo de las variedades Negrito y Chalqueño que alcanzaron hasta 5.7 y $5.5 \mathrm{~cm}$ día ${ }^{-1}$ planta $^{-1}$, respectivamente (Figura 3). El criollo Mején al presentar inicialmente una mayor altura de plántula (11 dds) podría tener ventajas ambientales en épocas donde la disponibilidad de agua es limitada (Karmore y Tomar, 2015).

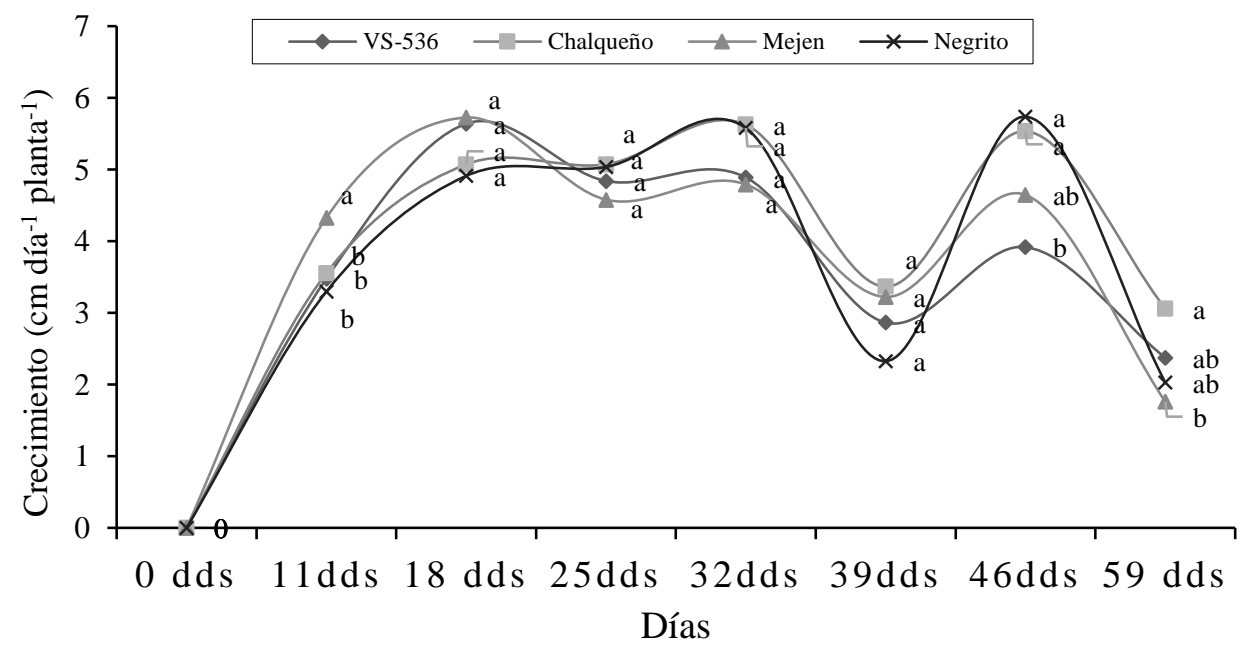

Figura 3. Tasa absoluta de crecimiento $\left(\mathrm{cm} \mathrm{día}^{-1}\right.$ planta $\left.^{-1}\right)$ de tres maíces criollos y una variedad sintética bajo tratamientos de osmoacondicionamiento (dds= días después de la siembra). 
La reducción de la tasa de crecimiento en el maíz Mején hacia el final del ciclo (59 dds) podría obedecer a su precocidad de floración, ya que en esas fechas inició la etapa de floración en esta variedad. Todos los maíces estudiados presentaron variaciones de la TAC a lo largo de su ciclo fenológico, pero a partir de los 46 dds el criollo Negrito sobresalió con la mayor tasa de crecimiento $\left(5.7 \mathrm{~cm} \mathrm{día}^{-1}\right.$ planta $\left.^{-1}\right)$ misma que se caracteriza por su alto porte y crecimiento rápido (CONABIO, 2010). Hacia la mitad del ciclo los criollos presentaron mayor tasa de crecimiento que la variedad VS-536, lo que indica que aquellas respondieron mejor a las condiciones ambientales (Figura 3).

\section{Tasa absoluta de crecimiento en materia seca}

El criollo Chalqueño a los 25 dds fue el que obtuvo una mayor ganancia de materia seca con 0.71 $\mathrm{g} \mathrm{día}^{-1}$ planta $^{-1}$ (Figura 4), lo que indica un crecimiento inicial rápido. Sin embargo, a los 39 dds el criollo que presentó mayor ganancia de materia seca fue Mején ( $1.11 \mathrm{~g} \mathrm{día}^{-1}$ planta $\left.^{-1}\right)$. A los 59 dds, los cuatro maíces mostraron una ganancia similar en materia seca. En esta etapa las plantas ya estaban en floración, aun así, la ganancia diaria de peso continuó en las cuatro variedades de maíz.

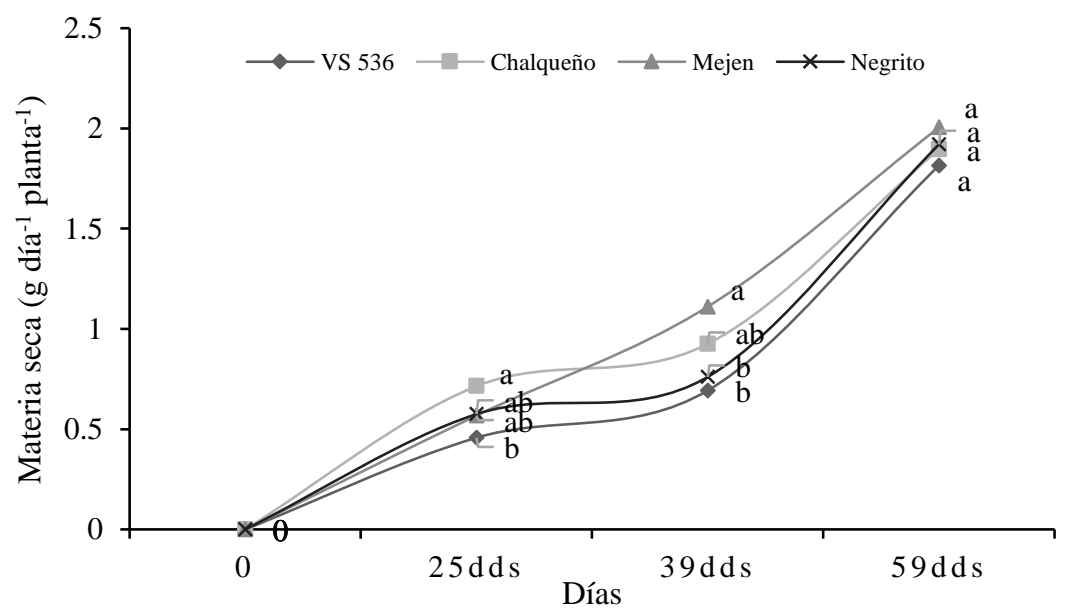

Figura 4. Ganancia de materia seca (g día ${ }^{-1}$ planta $^{-1}$ ) en tres maíces criollos y una variedad sintética bajo tratamientos de osmoacondicionamiento.

\section{Interacción tiempo*solución}

Los tratamientos agua $12 \mathrm{~h}, 18 \mathrm{~h}$ y $\mathrm{AG}_{3} 18 \mathrm{~h}$ indujeron mayor ganancia de materia seca $(0.64 \mathrm{~g}$ día $^{-1}$ planta $\left.^{-1}\right)$ a los $25 \mathrm{dds}$, en comparación con el testigo $\left(0.42 \mathrm{~g} \mathrm{día}^{-1}\right.$ planta $\left.^{-1}\right)$. Posteriormente (59 dds) las plantas resultantes del tratamiento agua $18 \mathrm{~h}$ mostraron considerablemente mayor ganancia (2.2 $\mathrm{g} \mathrm{día}^{-1}$ planta $\left.^{-1}\right)$ que el testigo (1.7 $\mathrm{g} \mathrm{día}^{-1}$ planta $\left.^{-1}\right)$. A lo largo del experimento, se encontró que el testigo produjo la menor tasa de ganancia de materia seca, por lo que se concluye que los tratamientos de osmoacondicionamiento inducen mayor producción de materia seca que los de semillas no tratadas (Figura 5).

La aplicación de tratamientos de osmoacondicionamiento favorece una mayor ganancia de materia seca por día, lo que favorecería mayor producción y rendimiento del cultivo. Lo encontrado en este trabajo coincide con lo reportado por Haider et al., (2016) en trigo, quienes reportan mayor rendimiento con los tratamientos de osmoacondicionamiento. En la literatura científica no se encontraron trabajos que reporten valores de la tasa absoluta de crecimiento para el maíz. 


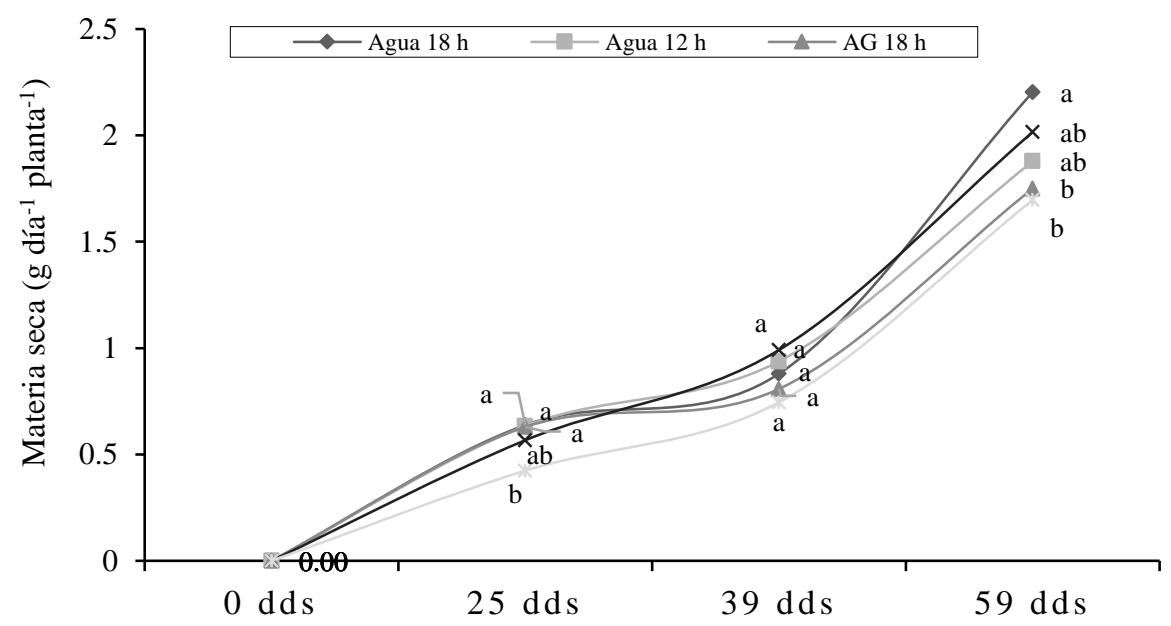

Figura 5. Efecto de la interacción solución *tiempo sobre la ganancia de materia seca ( $\mathrm{g} \mathrm{día}^{-1}$ planta $^{-1}$ ) en maíz sometido a tratamientos de osmoacondicionamiento.

\section{Días a floración masculina y femenina}

La floración mostró diferencias significativas entre variedades, siendo Mején la que alcanzó la floración masculina y femenina en menor tiempo (48 días y 55 días respectivamente). En contraste, las razas Negrito y Chalqueño tomaron más tiempo para alcanzar la etapa de floración (Figura 6). Resultados similares fueron publicados por De la Cruz-Lazaro et al. (2009) para la variedad VS536 a los 55 días después de la siembra.

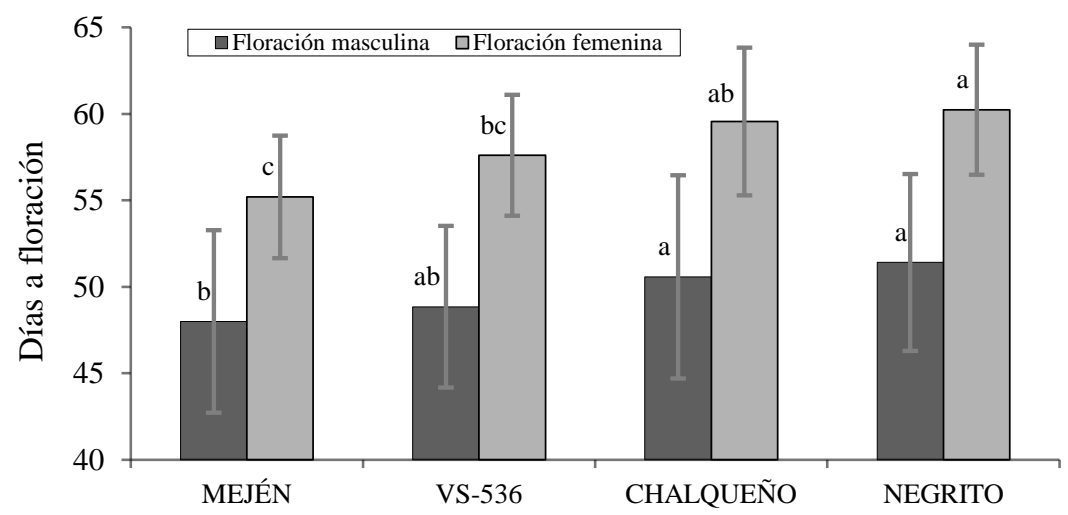

Figura 6. Efecto de tratamientos de osmoacondicionamiento sobre los días a floración en tres maíces criollos y una variedad sintética.

Los tratamientos de osmoacondicionamiento agua y $\mathrm{AG}_{3}$ lograron acortar los días a la floración masculina y femenina en comparación con el testigo. Esta precocidad fue de hasta cinco días para la primera y cuatro días para la floración femenina (Figura 7). Los tratamientos de osmoacondicionamiento de las semillas de maíz antes de la siembra resultan favorables ya que se podría obtener una cosecha más temprana en comparación con la siembra sin osmoacondicionamiento. En trabajos previos Mahboob et al. (2015) reportan que las plantas resultantes de semillas osmoacondicionadas presentan menor número de días a la madurez que las semillas no tratadas. 


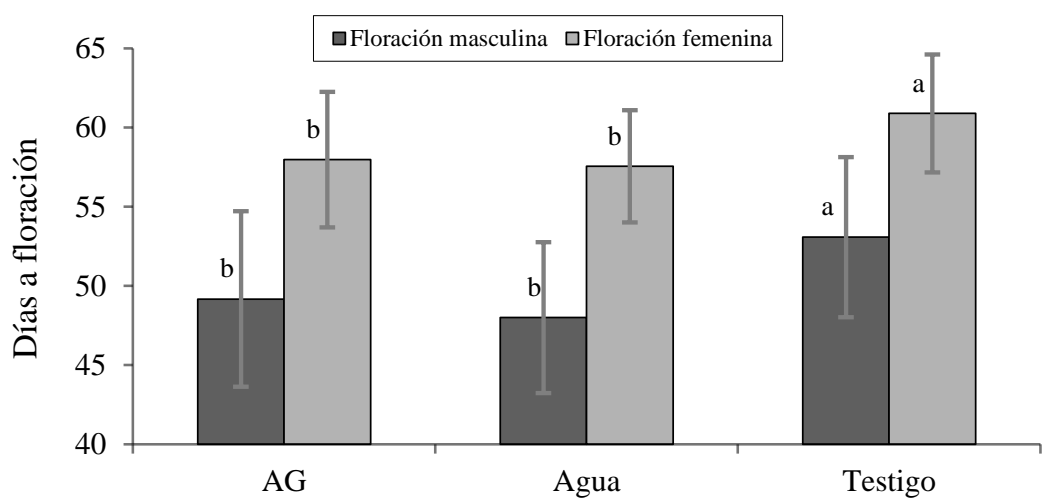

Figura 7. Días a floración masculina y femenina de plantas de maíz resultantes de semillas bajo diferentes tratamientos de osmoacondicionamiento.

\section{Interacción variedad*tiempo*solución}

En el análisis de la interacción variedad*tiempo*solución, se encontró que los tratamientos mostraron diferencias estadísticas significativas entre sí y respecto al testigo. Los tratamientos Chalqueño con agua por $18 \mathrm{~h}$ y Mején con $\mathrm{AG}_{3}$ por $18 \mathrm{~h}$, son los que presentaron el menor número de días a floración masculina (43 dds) mientras que el testigo Chalqueño tardó más días para llegar a floración masculina (57 dds). Por lo tanto, el uso de estas variedades en combinación con tiempo y solución osmótica acortaron el ciclo del cultivo; todos los tratamientos resultaron mejores que los testigos sin osmoacondicionamiento (Figura 8).
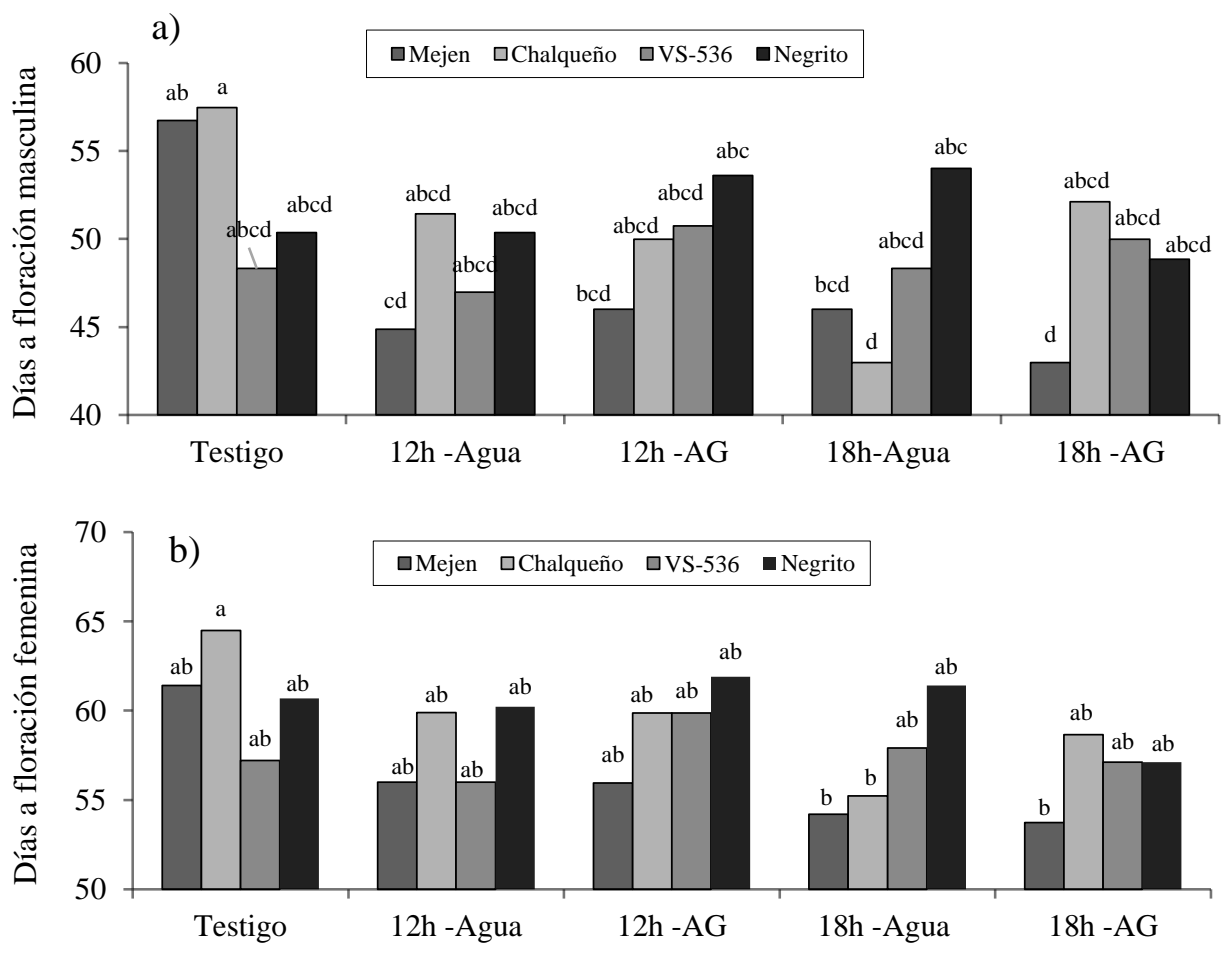

Figura 8. Días a floración masculina (a) y femenina (b) de plantas de maíz resultantes de semillas sometidas a tratamientos de osmoacondicionamiento. Literales diferentes dentro de la gráfica, indican diferencia estadística significativa. 
En cuanto a la floración femenina, la variedad Mején 18 h, lo realizó en 54 días mientras que Chalqueño testigo tardó 64 días para alcanzar esta floración. Se observó que el osmoacondicionamiento de las semillas de maíces criollos acorta el número de días a la floración femenina. Arif et al. (2014) indican que en soya los tratamientos de osmoacondicionamiento incrementaron la precocidad a la floración en comparación con el testigo. En este sentido, el presente trabajo es novedoso al incluir esta variable de respuesta a los tratamientos de osmoacondicionamiento ya que no se encontraron reportes previos al respecto en la literatura.

\section{Concentración de nitrógeno, fósforo y potasio en planta}

Los resultados de este trabajo muestran que la variedad VS-536 presentó las mayores concentraciones de nitrógeno $(1.62 \%)$, fósforo $(0.32 \%)$ y potasio $(0.57 \%)$, mientras que la variedad Negrito presentó los valores más bajos de estos elementos (1.07, 0.2 y 0.37, respectivamente), siendo estas comparaciones estadísticamente diferentes (Figura 9). Estos valores corresponden con el comportamiento de otras variables como altura y producción de materia seca.

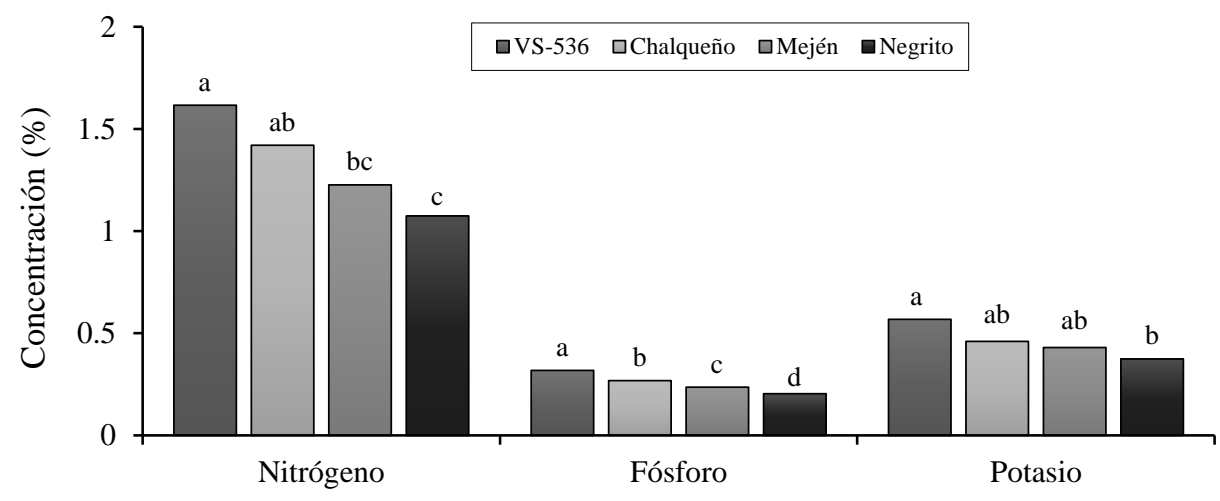

Figura 9. Concentración de nitrógeno, fósforo y potasio en cuatro variedades de maíz bajo tratamientos de osmoacondicionamiento.

A diferencia de lo anterior, los tiempos y solución de osmoacondicionamiento no influyeron en la concentración de NPK en la planta. Resultados similares fueron reportados por Bismillah et al. (2014) quienes no encontraron diferencia estadística significativa en el cultivo de trigo al usar agua en comparación con el testigo, sobre la concentración de fósforo y potasio en la planta. Para el caso de maíz bajo osmoacondicionamiento, no se encontraron reportes en la literatura.

\section{Conclusiones}

Las variedades de maíz presentaron alto porcentaje y velocidad de emergencia en respuesta a los factores tiempo y solución; los criollos Chalqueño y Negrito, en ambos tiempos de imbibición presentaron mayor porcentaje de emergencia en menor tiempo, mientras que Mején presentó mayor altura en los primeros días de siembra. Los factores tiempo y solución de osmoacondicionamiento favorecieron mayor producción de materia seca cuando las semillas fueron osmoacondicionadas con agua por 18 h. La interacción con la variedad no presentó diferencias significativas. 
Respecto a días a la floración, las plantas provenientes de las semillas de Mején osmoacondicionadas con $\mathrm{AG}_{3}$ por $18 \mathrm{~h}$ y las de Chalqueño osmoacondicionadas con agua por 18 $\mathrm{h}$, resultaron más precoces al presentar el menor número de días a la floración masculina. La concentración de NPK en planta solo presentó diferencia significativa para el factor variedad, mientras que los factores tipo de solución y tiempo de osmoacondicionamiento no mostraron efecto sobre esta variable.

\section{Literatura citada}

Afzal, I.; Shahzad, M. B.; Ahmad, N.; Mumtaz, A. C.; Ejaz, A. W. and Khaliq, A. 2002. Effect of priming and growth regulator treatments on emergence and seedling growth of hybrid maize (Zea mays L.). Inter. J. Agric. Biol. 2(4):303-306.

Ahammad, K. U.; Rahman, M. and Ahmed, M. 2014. Effect of osmopriming on the emergence of maize (Zea mays L.) seedling. Bangladesh J. Agric. Res. 3(39):427-435.

Ángeles-Gaspar, E.; Ortiz-Torres, E.; López, A. P. y López-Romero, G. 2010. Caracterización y rendimiento de poblaciones de maíz nativas de Molcaxac, Puebla. Rev. Fitotec. Mex. 4(33):287-296.

Arif, M.; Jan, M. T.; Mian, I.; Khan, S.; Hollington, P. and Harris, D. 2014. Evaluating the impact of osmopriming varying with polyethylene glycol concentrations and durations on soybean. Inter. J. Agric. Biol. 2(16):359-364.

Barrera, J.; Suárez, D. y Melgarejo, L. M. 2010. Análisis de crecimiento en plantas. Experimentos de fisiología vegetal. Colombia. 25-38 pp.

Barrón-Freyre, S. 2008. Manual para la producción de maíz en Tabasco. Huimanguillo, Tabasco. INIFAP. Folleto para productores núm. 13. 27 p. ISBN: 9786074250190.

Bismillah, M.; Gurchani, M. A.; Freed, S.; Jabran, K. and Hussain, M. 2014. Osmopriming improves the emergence, growth, nutrient uptake and soluble sugar contents of wheat seedlings. Soil Sci. Soc. Pak. 2(33):142-148.

CONABIO. 2010. Comisión Nacional para el Conocimiento y Uso de la Biodiversidad. Biodiversidad mexicana. http://www.biodiversidad.gob.mx/usos/maices/grupos/ chalqueno.html.

De la Cruz-Lazaro, E.; Córdova-Orellana, H.; Estrada-Botello, M.; Mendoza-Palacios, J.; GomezVazquez, A. y Brito-Manzano, N. 2009. Rendimiento de grano de genotipos de maíz sembrados bajo tres densidades de población. Universidad y Ciencia. 1(25):93-98.

Espinosa-Carderón, A.; Tadeo-Robledo, M.; Mora-García, K. y Turrent-Fernández, A. 2017. La libre circulación de las semillas y la agrodiversidad están en riesgo. Políticas públicas para un México megadiverso y pluricultural. 14-15 p.

Haider, M. U.; Hussain, M.; Khan, M. B.; Ijaz, M.; Sattar, A.; Akram, M. and Hassan,W. 2016. Influence of seed priming and seed size on wheat performance under different tillage systems. Inter. J. Agric. Biol. 18:858-864. DOI 10.17957/IJAB/15.018.

Imran, M.; Asim Mahmodd, V. R. and Neumann, G. 2013. Nutrient seed priming improves seedling development of maize exposed to low root zone temperatures during early growth. European J. Agron. 49:141-148. DOI 10.1016/j.eja.2013.04.001.

Karmore, J. V. and Tomar, G. 2015. Effects of seed priming methods on germination and seedling development of winter maize (Zea mays L.). Adv. Res. J. Crop. Improv. 2(6):88-93. 
Maguire, J. D. 1962. Speed of germination-aid selection and evaluation for seedling emergence selection and vigor. Crop Sci. 2:176-177. doi.org/10.2135/cropsci1962.0011183X0002000 20033x.

Mahboob, W.; Rehman, H. U.; Shahzad Maqsood Ahmad Basra, I. A.; Abbas, M. A. and Sarwar, M. N. 2015. Seed priming improves the performance of late sown spring maize (Zea mays) though better crop and physiological attributes. Inter. J. Agric. Biol. 17:491-498. DOI 10.17957/IJAB/17.3.14.283

Moradi-Dezfuli, P.; Sharif-Zadeh, F. and Janmohammadi, M. 2008. Influence of priming techniques on seed germination behavior of maize inbred lines (Zea mays). J. Agric. Biol. Sci. 3(3):22-25.

Nicasio-Arzeta, S.; Sanchez-Coronado, M. E.; Orozco-Segovia, A. y Gamboa-De Buen, A. 2011. Efecto del preacondicionamiento y el sustrato salino en la germinación y crecimiento de plantulas de maíz (Zea mays) raza Chalqueño. Agrociencia. 45(2):195-205.

Rangel-Fajardo, M. A.; Cordova-Tellez, L. y Cárdenas-Soriano, E. 2014. Pérdida de tolerancia a la desecación durante la imbibición-germinación de semillas de maíz. Rev. Mex. Cienc. Agríc. 5(5):833-845.

Tonaka, A. y Yamaguchi, J. 2014. Producción de materia seca, componentes del rendimiento y rendimiento del grano en maíz. $4^{\mathrm{a}}$ (Ed.). México: Colegio de Postgraduados en Ciencias Agrícolas. 87 p. ISBN: 9786077152262. 